\title{
Irritable bowel syndrome, depression and anxiety
}

\author{
Cafer Alhan ${ }^{1^{*}}$ and Aslıhan Okan ${ }^{2}$ \\ ${ }^{1}$ Department of Psychiatry, The Ministry of Health, Health Sciences University, Van Training and Research Hospital, Van, \\ Turkey \\ 2Department of Psychiatry, Dicle University, School of Medical, Diyarbakır, Turkey
}

\begin{abstract}
The relationship between psychiatric disorders and irritable bowel syndrome has been known for a long time. Although Irritable bowel syndrome (IBS) is a very common functional bowel disease associated with psychiatric disorders, its pathophysiology is not fully elucidated. Brain and bowel set up a bidirectional communication over the autonomic nervous system (ANS) and hypothalamic - pituitary - adrenal (HPA) axis. In a significant number of patients diagnosed with major depressive disorder (MDD) and anxiety disorders meet IBS criteria.
\end{abstract}

Key Words: Functional bowel disease, Irritable bowel syndrome, psychiatric disorders

\section{Introduction}

Effects of psychological situations on stomach and bowel have been known for centuries. Although functional gastrointestinal disorders are digestive system complaints, they are the disorders without structural (radiological, endoscopic) and biochemical abnormalities that explain chronic and recurrent gastrointestinal (GI) symptoms (1). Functional gastrointestinal disorders are common both in general population and clinical applications. In a population-based study in Western countries, it shows prevalence between $10-20 \% \quad(1,2)$. Especially in functional gastrointestinal patients with severe or refractory symptoms, the frequency of psychiatric diagnosis is between $42 \%$ and $61 \%$. Anxiety disorders, mood disorders (major depression) and somatoform disorders such as pain and somatization disorder can be considered among the most common diagnosis for the patients in reception centers (3).

The situations defined as "die of boredom" and "to soil one's pants out of fright" in daily use show how emotions are closely associated with GI tract (4). Beaumont is accepted to be the first person who experimentally investigated the effect of emotions on digestive organs. Beaumont, in an article published in 1833, stated that bile flow of a patient with an open gastric fistula due to a war wound accelerated if irritated $(5,6)$. In the $1880 \mathrm{~s}$, William James and Carl Lange formulated an emotion theory independently from each other. The main idea of the theory, which was also supported by Damasio and still has an impact today, is in the form of which emotional stimuli automatically cause the physical changes, and the basic of sense of feeling is the feedback of these bodily changes to the brain $(7,8)$. Whilst considering the many mechanisms that can affect the GI symptoms of psychological processes, R. Schindler makes the following definition which is still quite concerned with our time: "Psychoneurosis of the GI tract are the disorders in organ function of GI tract as a result of participation of psychological factors in the absence of anatomical changes' (9).

The brain and bowel work in a highly integrated way and they set up a bidirectional communication largely over autonomic nervous system (ANS) and the hypothalamic - pituitary - adrenal (HPA) axis. In central nervous system (CNS), the focus of bowel control responsible for both interior and exterior homeostasis of the organism is mainly limbic system. The limbic system plays a central role for emotion; it is a nonverbal system which facilitates survival, threat avoidance, social interaction and learning. Emotion band and related physiological changes are also work of limbic system, and if looked at neuroanatomical perspective, "mind/body interaction" largely arises in this area. Indeed, the limbic system is involved in "top-down" modulation of visceral pain and visceral perception. This bidirectional 
communication includes various cognitive /psychological factors, visceral perception and motor abnormalities (10).

The pathophysiology of the functional gastrointestinal disease is not fully understood, and it is probably multifactorial. It was focused on stress and bowel hyperactivity in first period studies, but it was more focused on the endorgan motility or sensory impairment and harmony in subsequent studies. It has become more obvious that standard reductionist approach was not sufficient in pathophysiology, therefore broader, more integrated conceptualization is required. This conceptualization includes the sense of the relationship between gastrointestinal function and central nervous system, and it is known as the biopsychosocial model.

\section{Irritable Bowel Syndrome}

Irritable bowel syndrome is a chronic disorder characterized by abdominal pain, abdominal discomfort and changes in bowel habits, and without any/known organic causes leading to constipation and diarrhea (11). Spastic bowel disease is also known as spastic colitis or functional bowel disease (12). IBS is located between functional GI syndromes, and the prevalence varies between 3-20\% (13). Although IBS affects all age groups and both genders, majority of the people diagnosed is composed of young people and females (14). In a study to determine the prevalence of IBS in primary health care in Turkey, 598 (41\%) out of 1461 cases which were randomly selected from 2157 patients with gastrointestinal system (GIS) complaints for the survey and $141(19 \%)$ out of 742 cases which were randomly selected from 5363 patients with nonGIS complaints for the survey were diagnosed with IBS (15).

Upper GI tract symptoms including gastroesophageal reflux, dysphagia, early satiety, intermittent dyspepsia, nausea, non-cardiac chest pain are common in IBS patients (16). Also, extraintestinal complaints including sexual dysfunction, dysmenorrhea, dyspareunia, increase in urinary frequency and fibromyalgia symptoms are common in patients $(17,18)$. Most of these extraintestinal symptoms were widely reported as concomitant diseases for the patients with functional GI disorders such as IBS. Altered autonomic regulation and extraintestinal symptoms indicate a more general CNS disorders. Altered sleep patterns, especially altered REM sleep, may be a new evidence in IBS patients for the dysfunction of CNS-related with 5-HT (19). In addition, in treatments that target serotonergic transmission in the CNS, for example, the activity of antidepressants and anxiolytics has been shown in the treatment of symptoms associated with IBS (20). These findings show that changes in 5 - HT transmission may have a central role in brainbowel interactions, and it may play a role in IBS development or perception of the symptoms. However, it should not be thought that the "5HT hypothesis" is effective by itself. Balance is required between monoaminergic systems including adrenergic, serotonergic and dopaminergic systems. Autonomic dysfunction has been reported in patients with IBS, and therefore IBS may also represent one of the pathophysiological mechanisms of the intestinal and extraintestinal symptoms $(21,22)$.

Childhood experiences can affect psychological tendencies. Together with the reaching puberty, genetics, early learning, special and other environmental effects are integrated with each unique personality and behavior. These factors affect the determination of stress level and the ability of a person to handle these. Divorce, relationship difficulties, serious illnesses (self or others), failure at work, difficulties in harboring and dismissing with force are some part of the chronic life stresses. Perception of control, all seeking excitement status and psychosocial existence level on persons unresolved stressed life events could mediate to life stress (23).

\section{Irritable Bowel Syndrome and Depression}

It is known that changes occur in bowel habits, and even frequently IBS is seen in Major depressive disorder (MDD) (24). Tollefson et al. (25) reported that IBS criteria met among 30\% of the patients diagnosed with MDD and $11 \%$ of the control group (4). Masand et al. (26) similarly detected IBS frequency as $27 \%$ in MDD patients and $3 \%$ in control group. Again Masand et al. (27) in patients with major depressive disorder developed on dysthymia level (double depression) found IBS frequency as 58\%, in control group ratio was limited to $3 \%$. Also in patients diagnosed with IBS, MDD additional diagnosis is very common. Mayer et al. (28) with Creed stated that, IBS in early stages is associated with anxiety and in the long term with depression (29). Against the epidemiological data at hand, IBS co-occurring with depression and anxiety disorders cause patients search for help more frequently and due to this properties of the patients included in the 
study it is suggested that detected ratios could be misleading $(29,30)$. Thus, it is claimed that more than $50 \%$ of the patients who are looking for treatment due to IBS, had depression and anxiety disorder (31).

It can be thought that being of IBS is painful, and chronic disease plays role in the frequent cooccurrence of IBD with MDD. However, Henningsen et al. (32) in a very detailed metaanalysis in which they investigated that relationship of 4 somatic functional disorders (IBS, non-ulcer dyspepsia, fibromyalgia and chronic fatigue syndrome) including IBS with anxiety and depression, stated that this opinion may not be true. They claimed that somatoform disorders, in which they claim IBS is included, are some type of internal perception disorder and caused by a disorder in the perception of bodies physiological state. Clinical evidence about the close relation of IBS and depression, give rise to thought that pathophysiological irregularities underlying these two disorders intersect at one point (4). It is known that in many IBS patient's mucosa there are elevated activated immune system cells, and inflammation is accompanying to IBS although at low levels (33). Since some mediators secreted from mast cells can change neuronal and muscle functions, these cells became very important candidates for the pathophysiology of IBS. In an important study carried out by Piche et al. (34) IBS and depression relation at bowel mucosa level was tried to be elucidated. Mucosa samples were compared in IBS patients, healthy individuals and depressive patients without IBS complaints included in the study. Inflammatory changes at low levels in IBS patients, the relation of mast cell number with the severity of exhaustion and depressive complaints were shown. Together with this, bowel mucosa in depression patients without IBS complaints has been found to be not different from healthy controls in terms of inflammation. In animal models of stress, it is shown that it can trigger the inflammation response in GI system and increase intestinal permeability $(35,36)$. In addition to depression triggering inflammation, it is known that inflammatory mediators could intensify depression, cytokine levels are increased in depression (37). In spite of this, in a study of Piche et al. (34) not seeing inflammation in colon mucosa in patients only with depression and contrary to this seeing inflammation in IBS patients with depressive complaints, interpreted as stress alone is not sufficient to trigger inflammation. Thus in a mouse study carried out by Varghese et al. (38) Similar results were obtained, and susceptibility to inflammation could be improved with antidepressants.

\section{Irritable Bowel Syndrome and Anxiety Disorders}

Anxiety disorders are comorbid diseases seen very commonly in IBS patients. Garakani et al. (24) investigated the relations between anxiety disorders and IBS and found a powerful connection between symptoms and presentations. In $44 \%$ of patients with panic disorder, both diseases variety of symptom characteristics like abdominal pain, diarrhea, and nausea have been found together with comorbid IBS (39). IBS has been found in a high frequency of $35 \%$ in obsessive-compulsive disorder (OCD) and 37\% in common anxiety disorder (CAD). Similarly in IBD patients, including post-traumatic stress disorder $36 \%$, comorbid anxiety disorders have been found in a high frequency (40). Together with this, our knowledge about the relation between specific anxiety disorders and IBS is still limited due to high comorbidity seen with anxiety disorders. In an investigation about the comorbidity ratios in anxiety and depressive disorders, 55\% of the patients diagnosed with anxiety disorder reported symptoms meet at least one additional anxiety or depressive disorder criteria during the evaluation time (41). When life-long diagnosis are considered comorbidity ratio elevates to $76 \%$.

Therefore due to the limited coverage of previous studies on just one isolated anxiety disorder and IBS, when all anxiety disorders were considered it is difficult to determine genuine relations. For example in a study about rectal distension low tolerance has been found to be related to PB and total somatic symptoms, however, not to be related to anxiety or depression (42). Similarly, high comorbidity ratio between anxiety disorders and IBS can be stimulated by both IBS and all anxiety disorders with from one or two anxiety disorders (for example $\mathrm{PB}$ and CAD) with the high comorbid ratio.

In a conclusion, the association of IBS and psychiatric disorders has long been known. Together with IBS especially anxiety disorders and depression occurrence is frequently seen. It is clear that a multidisciplinary approach to patients with IBS approach and a follow up will contribute positively. If clinicians only focus on the symptoms of IBS and cannot see the patient as a biopsychosocial whole, they will be insufficient to provide successful treatment for the disease.

East J Med Volume:22, Number:3, July-September/2017 
Besides, diagnosis of IBS by psychiatrists will make serious contribution to the treatment of Depression and Anxiety disorders.

\section{References}

1. Mungan Z. Fonksiyonel sindirim sistemi hastalıklarına yaklaşım. 6. Ulusal İç Hastalıkları Kongresi, Göynük/ Antalya, 14-19 Eylül 2004.

2. Chang L. Review article: epidemiology and quality of life in functional gastrointestinal disorders. Aliment Pharmacol Ther 2004; 20: 31-39.

3. Jones MP, Crowell MD, Olden KW, Creed F. Functional gastrointestinal disorders: an update the frequency of irritable bowel syndrome (IBS) in primary care centers of Turkeythe frequency of irritable bowel syndrome (IBS) in primary care centers of Turkeythe frequency of irritable bowel syndrome (IBS) in primary care centers of Turkeythe frequency of irritable bowel syndrome (IBS) in primary care centers of Turkeythe frequency of irritable bowel syndrome (IBS) in primary care centers of Turkeyfor the psychiatrist. Psychosomatics 2007; 48: 93-102.

4. Eker MÇ, Eker ÖD. Neurobiology of Depression and Irritable Bowel Syndrome Comorbidity, Current Approaches in Psychiatry 2009; 1: 120-131.

5. Beaumont W. Experiments and Observations on the Gastric Juice and the Physiology of Digestion. Plattsburgh: F. P. Allen, 1833.

6. Musial F, Häuser W, Langhorst J, Dobos G, Enck P. Psychophysiology of visceral pain in IBS and health. J Psychosom Res 2008; 64: 589-597.

7. James W. What is an emotion? Mind 1884; 9: 188205.

8. Porges SW. The polyvagal perspective. Biol Psychol 2007; 74: 116-143.

9. Van Oudenhove L, Vandenberghe J, Demyttenaere K, Tack J. Psychosocial factors, psychiatric illness and functional gastrointestinal disorders: a historical perspective. Digestion 2010; 82: 201-210.

10. Jones MP, Dilley JB, Drossman D, Crowell MD. Brain-gut connections in functional GI disorders: anatomic and physiologic relationships. Neurogastroenterol Motil 2006; 18: 91-103.

11. Grundmann O, Yoon SL. Irritable bowel syndrome: epidemiology, diagnosis and treatment: an update for health-care practitioners. J Gastroenterol Hepatol 2010; 25: 691-699.

12. Yılmaz $S_{s}$ Dursun M, Ertem M, Canoruç F, Turhanoğlu A. The epidemiological aspects of irritable bowel syndrome in Southeastern Anatolia: a stratified randomised community based study. Int J Clin Pract 2005; 3: 361-369.

13. Drossman DA, Camilleri M, Mayer EA, Whitehead WE. AGA technical review on irritable bowel syndrome. Gastroenterology 2002; 123: 2108-2131.

14. Saito YA, Schoenfeld P, Locke GR 3rd. The epidemiology of irritable bowel syndrome in North America: a systematic review. Am J Gastroenterol 2002; 97: 1910-1915.

15. Özden A, Köksal AS, Oğuz D, et al. The frequency of irritable bowel syndrome (IBS) in primary care centers of Turkey. J Academic Gastroenterology 2006; 5: 4-14.

16. Longstreth GF, Thompson WG, Chey WD, et al. Functional bowel disorders. Gastroenterology 2006; 130: 1480-1491.

17. Whorwell PJ, McCallum M, Creed FH, Roberts CT. Non-colonic features of irritable bowel syndrome. Gut 1986; 27: 37-40.

18. Hershfield NB. Nongastrointestinal symptoms of irritable bowel syndrome: an officebased clinical survey. Can J Gastroenterol 2005; 19: 231-234.

19. Orr WC, Crowell MD, Lin B, Harnish MJ, Chen JD. Sleep and gastric function in irritable bowel syndrome: derailing the brain-gut axis. Gut 1997; 41: 390-393.

20. Cremonini F, Talley NJ. Diagnostic and therapeutic strategies in the irritable bowel syndrome. Minerva Med 2004; 95: 427-441.

21. Jarrett ME, Burr RL, Cain KC, et al. Anxiety and depression are related to autonomic nervous system function in women with irritable bowel syndrome. Dig Dis Sci 2003; 48: 386-394.

22. Jones MP, Crowell MD, Olden KW, Creed F. Functional gastrointestinal disorders: an update for the psychiatrist. Psychosomatics 2007; 48: 93102.

23. Sarason IG, Johnson JH, Siegel JM. Assessing the impact of life changes: development of the Life Experiences Survey. J Consult Clin Psychol 1978; 46: 932-946.

24. Garakani A, Win T, Virk S, et al. Comorbidity of irritable bowel syndrome in psychiatric patients: a review. Am J Ther 2003; 10: 61-67.

25. Tollefson GD, Tollefson SL, Pederson M. Comorbid irritable bowel syndrome in patients with generalized anxiety disorder and major depression. Ann Clin Psychiatry 1991; 3: 215-222.

26. Masand PS, Kaplan DS, Gupta S, et al. Major depression and irritable bowel syndrome: is there a relationship. J Clin Psychiatry 1995; 56: 363365.

27. Masand PS, Kaplan D, Gupta S, Bhandary AN. The relationship between irritable bowel syndromeand double depression (dysthymia and major depression). Depression 1995; 3: 303-308.

28. Mayer EA, Craske M, Naliboff BD. Depression, anxiety, and the gastrointestinal system. J Clin Psychiatry 2001; 62: 28-36. 
29. Creed F. The relationship between psychosocial parameters and outcome in irritable bowel syndrome. Am J Med 1999; 107: 74-80.

30. Talley NJ, Spiller R. Irritabl Bowel Syndrome: A poorly understood organic disease? Lancet 2002; 360: 555-564.

31. Drossman DA. The functional gastrointestinal disorders and the Rome III process. Gastroenterology 2006; 130: 1377-1390.

32. Henningsen $P$, Zimmermann $T$, Sattel $H$. Medically unexplained physical symptoms, anxiety, and depression: a meta - analytic review. Psychosom Med 2003; 65: 528-533.

33. Lydiard RB. Irritabl bowel syndrome, anxiety, and depression: what are the links? J Clin Psychiatry 2001; 62: 38-45.

34. Piche T, Saint-Paul MC, Dainese R, et al. Mast cells and cellularity of the colonic mucosa correlated with fatigue and depression in irritable bowel syndrome. Gut 2008; 57: 468-473.

35. Qiu BS, Vallance BA, Blennerhassett PA, Collins $\mathrm{SM}$. The role of $\mathrm{CD} 4+$ lymphocytes in the susceptibility of mice to stress-induced reactivation of experimental colitis. Nat Med 1999; 5: 1178-1182.

36. Kiliaan AJ, Saunders PR, Bijlsma PB, et al. Stress stimulates transepithelial macromolecular uptake in rat jejunum. Am J Physiol 1998; 275: 1037 1044.
37. Khairova RA, Machado-Vieira R, Du J, Manji HK. A potential role for proinflammatory cytokines in regulating synaptic plasticity in major depressive disorder.Int J Neuropsychopharmacol 2009; 12: 561-578.

38. Varghese AK, Verdú EF, Bercik P, et al. Antidepressants attenuate increased susceptibility to colitis in a murine model of depression. Gastroenterology 2006; 130: 1743-1753.

39. Gros DF, Antony MM, McCabe RE, Swinson RP. Frequency and severity of the symptoms of irritable bowel syndrome across the anxiety disorders and depression.J Anxiety Disord 2009; 23: 290-296.

40. Irwin C, Falsetti SA, Lydiard RB, et al. Comorbidity of posttraumatic stress disorder and irritable bowel syndrome. J Clin Psychiatry 1996; 57: 576-578.

41. Brown TA and Barlow DH. Classification of anxiety and mood disorders. In: D. H. Barlow (Ed.), Anxiety and its disorders: the nature and treatment of anxiety and panic New York: Guilford Pres. 2002; (2nd ed. pp. 292 - 327).

42. Guthrie E, Barlow J, Fernandes L, et al. Changes in tolerance to rectal distension correlate with changes in psychological state in patients with severe irritable bowel syndrome. Psychosomatic Medicine 2004; 66: 578-582. 